\title{
Outcomes variation in weight and resolution of obesity co-morbidities between five bariatric operations
}

\begin{abstract}
Purpose: Several bariatric operations are utilized to treat the growing obesity epidemic. The goal of this study was to identify variations in post-operative weight loss and obesityrelated co-morbidities between the most commonly performed weight loss operations.

Methods: Data from 166,601 patients in the Surgical Review Corporation's Bariatric Outcomes Longitudinal Database (BOLD) who underwent Laparoscopic Adjustable Gastric Banding (LAGB, $\mathrm{n}=67,514)$, Bilio-Pancreatic Diversion/Duodenal Switch (BPD/ DS, $n=1,673$ ), laparoscopic (LRYGB, $n=83,059$ ) and open (RYGB, $n=5,389$ ) Roux-en-Y gastric bypass, and Laparoscopic Sleeve Gastrectomy (SG, n=8966) was analyzed at baseline, and at 2, 4, 6, 12, 18, and 24 months post-operatively. Weight, BMI, and 29 weight related medical conditions were analyzed.
\end{abstract}

Statistics: Continuous variables were analyzed using ANOVA with treatment in the model. General Linear Models included baseline and post-operative data, modified for binomial distribution of dichotomous variables.

Results: BPD/DS patients had the lowest weight at 24 months and highest resolution of hypertension, diabetes, hyperlipidemia, and asthma, but most increase in cholelithiasis, liver disease, abdominal hernias, and psychological disorders. LRYGB best treated GERD and somatic disorders. OSA and gout resolved most after SG. LAGB was least effective in weight loss and co-morbidity resolution, but least likely to develop post-op liver disease, cholelithiasis, abdominal hernias, and symptomatic abdominal skin.

Conclusions: Post-operative weight loss and rates of obesity-related co-morbidities vary between LAGB, BPD/DS, RYGB, LRYGB, and SG. Knowledge of these differences can help guide the pre-operative evaluation of obese patients presenting for weight loss surgery.

Keywords: obesity, laparoscopic, sleeve gastrectomy, gastric
Volume 10 Issue 4 - 2020

\author{
Christopher Finley, Leon Kushnir, Gus J \\ Slotman \\ Inspira Health Network, USA
}

Correspondence: Gus J Slotman, Director of Clinical Research, Inspira Health Network, Clinical Professor of Surgery Rowan SOM, I 505 W Sherman Avenue Suite B,Vineland, NJ 08360, USA, Tel 856-64I-8635, Fax 856-64I-8636, Email Slotang@ihn.org

Received: July 21, 2020 | Published: August 21, 2020
Abbreviations: LAGB, laparoscopic adjustable gastric banding; BPD/DS, bilio-pancreatic diversion/duodenal switch; LRYGB, laparoscopic roux-en-Ygastric bypass; SG, sleeve gastrectomy

\section{Introduction}

Given the limited success of medical weight loss regimens in treating complicated morbidly obese patients, ${ }^{1-4}$ bariatric surgery continues to serve as the most effective modality for weight loss in these patients. ${ }^{5}$ With several surgical procedures available, matching each individual patient with the most appropriate surgery continues to evolve.At present, patient and/or surgeon preferences currently drive the selection of which bariatric surgery to employ for a patient. ${ }^{6}$ Extensive investigations into the short- and long-term efficacies of and between each bariatric procedure continue to reveal varying advantages for each modality. Currently, the two surgeries being performed most frequently are SG and RYGB/LRYGB.$^{7,8}$ Recent investigations have not demonstrated consistent differences in weight loss between these two procedures, but outcomes in resolution of comorbidities favors gastric bypass. ${ }^{9-12} \mathrm{BPD} / \mathrm{DS}$ provides added weight loss and resolution of co-morbidities compared with RYGB. ${ }^{13,14}$ However, few investigations have evaluated baseline pre-operative clinical characteristics and long-term outcomes for weight and weight-related medical problems, comparing the results of all five operations. The objective of this study was to compare post-operative weight, BMI, and rates of obesity-related co-morbidities for up to 24 months after LAGB, BPD/DS, RYGB, LYRGB, and SG.

\section{Methods}

The investigation was approved by the Data Access Committee of the Surgical Review Corporation and by the IRB of Our Lady of Lourdes Medical Center, Camden, NJ. Data from 166,601 patients in the Surgical Review Corporation's Bariatrics Outcomes Longitudinal Database (BOLD) $)^{15}$ who underwent LAGB $(n=67,514)$, BPD/DS $(\mathrm{n}=1,673)$, LRYGB $(\mathrm{n}=83,059), \operatorname{RYGB}(5,389)$, and SG $(\mathrm{n}=8966)$ as the first bariatric operation and recorded at least one post-operative visit was analyzed at baseline and at 2, 4, 6, 12, 18, and 24 months post-operatively from June 1, 2007 to December 31, 2010. Weight, BMI, and 29 obesity-related health conditions were analyzed. Diagnosis of co-morbidities followed BOLD criteria and included cardiopulmonary, metabolic, endocrine, hepatobiliary, abdominal, somatic, psychological, and behavioral disorders.

\section{Statistical analysis}

Continuous variables were analyzed using an ANOVA with baseline and treatment in the model. Pair-wise comparisons were performed for continuous variables on the least squares means of the treatments calculated from the ANOVA model to find differences 
in the treatment groups. Distribution of weight, BMI, and obesity co-morbidities was examined by using a general linear model with baseline and treatment in the model and modified for a binomial distribution to account for the co- morbidities being dichotomous variables. Pairwise comparisons were made for weight, BMI, and each set of comorbidities. ${ }^{16}$

\section{Results}

Post-operative weight, BMI, and number of patients at each data point are tabulated in Table 1. The five patient groups did not vary in age or in sex distribution. BPD/DS patients presented with the highest BMI at baseline and then were lowest at 24 months. RYGB had the second highest BMI at baseline and second lowest at 24 months. LYRGB and SG BMI's were intermediate between BPD/DS and RYGB versus LAGB both at baseline and in follow-up. LAGB BMI was lowest pre-operatively and highest at 24 months. Cardiopulmonary disease outcomes are displayed in Table 2. LAGB: Hypertension, CHF, angina, PVD, asthma, pulmonary hypertension, OSA, and obesity hypoventilation syndrome were lowest pre-operatively but persisted at highest or second highest rates at 24 months. BPD/DS: Hypertension and asthma were highest at baseline and lowest at 24 months. BPD/DS patients suffered from angina, CHF, pulmonary hypertension, and OSA most commonly at baseline and continued to have the highest frequency of these diseases post-operatively. PVD and Obesity Hypoventilation Syndrome were not seen in BPD/DS patients by 24 months. LRYGB: Asthma was lowest post-operatively. Hypertension, OSA, and Obesity Hypoventilation were second lowest. RYGB: Hypertension, angina, asthma, and CHF were second most common post-operatively with the lowest relative reduction from baseline. OSA and PVD were most frequent post-operatively. Obesity Hypoventilation Syndrome was second lowest. SG: Patients in SG group had the second lowest frequency of post-operative hypertension and least angina, CHF, OSA, and pulmonary hypertension. Postoperative rates of Obesity Hypoventilation Syndrome and asthma rates were highest after SG. Table 3 demonstrates outcomes in metabolic and endocrine disorders. LAGB: Baseline rates of diabetes and hyperlipidemia were lowest/second, respectively, but were highest by 24 months. BPD/DS: Diabetes and hyperlipidemia were highest at baseline and lowest at 24 months. BPD/DS patients also had the lowest pseudotumor cerebri. Postoperative gout and PCOS were highest 24 months after BPD/DS. LRYGB: Pseudotumor cerebri was highest postoperatively, and gout and hyperlipidemia were second lowest. RYGB: The second highest rates of postoperative diabetes, gout, hyperlipidemia, and pseudotumor cerebri were seen in the RYGB group. SG: Post-operative gout and PCOS were least frequent in these patients and diabetes was second lowest.

Table I Patient demographics, baseline weight, and post-operative weight loss and BMI

\begin{tabular}{|c|c|c|c|c|c|c|c|}
\hline \multicolumn{8}{|c|}{ Demographics, weight, and BMI } \\
\hline & Months: & Baseline & 2 & 6 & 12 & 18 & 24 \\
\hline & \multicolumn{7}{|l|}{ Operation } \\
\hline & LAGB & 67514 & 65866 & 46324 & $2664 \mid$ & 12323 & 8349 \\
\hline Number & $\mathrm{BPD} / \mathrm{DS}$ & 1673 & 1624 & 1049 & 619 & 234 & $|4|$ \\
\hline of patients & LRYGB & 83059 & 80933 & 46496 & $26|3|$ & 6826 & 5362 \\
\hline at each & RYGB & 5389 & 5164 & 3353 & 2092 & 823 & 692 \\
\hline \multirow[t]{3}{*}{ Interval } & SG & 8966 & 8797 & 3522 & 1382 & 325 & 164 \\
\hline & LAGB & $125 \pm 24$ & $119 \pm 18$ & $\mid 12 \pm \|$ & $107 \pm 11$ & $103 \pm 14$ & $102 \pm 14$ \\
\hline & BPD/DS & $149 \pm 33$ & $112 \pm 18$ & $92 \pm 9$ & $77 \pm 11$ & $72 \pm 13$ & $71 \pm 14$ \\
\hline \multirow[t]{6}{*}{ Weight (kg) } & LRYGB & $133 \pm 27$ & $113 \pm 20$ & $95 \pm 9$ & $84 \pm 11$ & $81 \pm 13$ & $82 \pm 14$ \\
\hline & RYGB & $|4| \pm 34$ & $113 \pm 18$ & $95 \pm 9$ & $85 \pm 11$ & $81 \pm 13$ & $80 \pm 14$ \\
\hline & SG & $134 \pm 32$ & $115 \pm 29$ & $99 \pm 9$ & $90 \pm 12$ & $88 \pm 14$ & $86 \pm 14$ \\
\hline & $P$ value & $<0.0001$ & $<0.0001$ & $<0.0001$ & $<0.0001$ & $<0.0001$ & $<0.0001$ \\
\hline & LAGB & $44.9 \pm 7$ & $42.7 \pm 3$ & $40.3 \pm 3$ & $38.4 \pm 4$ & $37.2 \pm 5$ & $36.8 \pm 5$ \\
\hline & BPD/DS & $52.1 \pm 10$ & $40.1 \pm 2$ & $33.0 \pm 3$ & $28.0 \pm 4$ & $26.2 \pm 5$ & $225.9 \pm 5$ \\
\hline \multirow[t]{4}{*}{ BMI } & LRYGB & $47.6 \pm 8$ & $40.6 \pm 3$ & $33.9 \pm 3$ & $30.3 \pm 4$ & $29.3 \pm 5$ & $29.5 \pm 5$ \\
\hline & RYGB & $50.2 \pm 10$ & $40.5 \pm 3$ & $34.0 \pm 3$ & $30.4 \pm 4$ & $29.2 \pm 5$ & $29.1 \pm 5$ \\
\hline & SG & $47.4 \pm 9$ & $41.0 \pm 3$ & $35.3 \pm 3$ & $32.8 \pm 4$ & $32.0 \pm 5$ & $31.4 \pm 5$ \\
\hline & $P$ value & $<0.0001$ & $<0.0001$ & $<0.0001$ & $<0.0001$ & $<0.0001$ & $<0.0001$ \\
\hline
\end{tabular}


Table 2 Cardiopulmonary baseline and post-operative outcomes by operation

\begin{tabular}{|c|c|c|c|c|c|c|c|}
\hline \multicolumn{8}{|c|}{ Cardiopulmonary co-morbidities (\%) } \\
\hline & Months: & Baseline & 2 & 6 & 12 & 18 & 24 \\
\hline & \multicolumn{7}{|l|}{ Operation } \\
\hline & LAGB & 55.3 & 51.71 & 48.38 & 45.43 & 43.85 & 41.24 \\
\hline & BPD/DS & 62.82 & 50.86 & 40.51 & 31.66 & 30.34 & 26.24 \\
\hline \multirow[t]{6}{*}{ Hypertension } & LRYGB & 60.4 & 49.85 & 39.71 & 33.32 & 30.3 & 28.65 \\
\hline & RYGB & 61.79 & 53.89 & 46.17 & 43.21 & 43.01 & 40.75 \\
\hline & SG & 55.01 & 47.46 & 41.17 & 35.53 & 34.46 & 28.66 \\
\hline & $\mathrm{P}$ value & $<0.0001$ & $<0.0001$ & $<0.0001$ & $<0.0001$ & $<0.0001$ & $<0.0001$ \\
\hline & LAGB & 2.16 & 1.86 & 1.58 & 1.46 & 1.62 & 1.5 \\
\hline & $\mathrm{BPD} / \mathrm{DS}$ & 3.59 & 2.59 & 2.67 & 2.26 & 2.99 & 2.84 \\
\hline \multirow[t]{5}{*}{ Angina } & LRYGB & 2.88 & 2.27 & 1.9 & 1.73 & I.7 & 1.59 \\
\hline & RYGB & 3.66 & 2.83 & 3.13 & 2.58 & 3.04 & 2.6 \\
\hline & SG & 2.48 & 2.13 & 1.65 & 1.66 & 1.85 & 0.61 \\
\hline & $\mathrm{P}$ value & $<0.0001$ & $<0.0001$ & $<0.0001$ & $<0.0001$ & 0.0225 & 0.1156 \\
\hline & LAGB & 1.56 & 1.45 & 1.38 & I.34 & I.4I & I.34 \\
\hline Congestive & BPD/DS & 4.18 & 5.23 & 5.24 & 4.04 & 4.7 & 4.96 \\
\hline Heart & LRYGB & 2.28 & 2.22 & 2.05 & 1.76 & 1.68 & 1.72 \\
\hline \multirow[t]{4}{*}{ Failure } & RYGB & 3.06 & 2.94 & 2.86 & 2.96 & 3.04 & 3.03 \\
\hline & SG & 1.94 & 1.89 & 1.99 & 1.81 & 1.85 & $0.6 I$ \\
\hline & $P$ value & $<0.0001$ & $<0.0001$ & $<0.0001$ & $<0.0001$ & $<0.0001$ & $<0.0001$ \\
\hline & LAGB & 0.95 & 0.91 & 0.88 & 0.95 & 1.03 & 0.93 \\
\hline Peripheral & BPD/DS & 2.33 & 1.54 & 1.24 & 1.29 & 0.43 & 0 \\
\hline Vascular & LRYGB & 1.19 & 1.09 & 0.96 & 0.95 & 0.89 & 0.95 \\
\hline \multirow[t]{5}{*}{ Disease } & RYGB & 1.54 & 1.37 & 1.46 & $\mathrm{I} .77$ & 1.94 & 1.59 \\
\hline & SG & 0.97 & 0.78 & 0.77 & 0.72 & I.54 & 1.22 \\
\hline & $P$ value & $<0.0001$ & $<0.0001$ & 0.0069 & 0.0037 & 0.0457 & 0.3544 \\
\hline & LAGB & 3.73 & 2.87 & 2.52 & 2.02 & 1.91 & 1.84 \\
\hline & BPD/DS & 12.67 & 12.25 & 10.77 & 9.21 & 7.69 & 2.13 \\
\hline Pulmonary & LRYGB & 4.93 & 3.58 & 2.03 & 1.39 & 1.25 & 1.21 \\
\hline \multirow[t]{4}{*}{ Hypertension } & RYGB & 4.12 & 3.33 & 1.82 & 1.43 & 0.73 & 1.16 \\
\hline & SG & 4.06 & 3 & 2.47 & 2.24 & 1.54 & 0.61 \\
\hline & $\mathrm{P}$ value & $<0.0001$ & $<0.0001$ & $<0.0001$ & $<0.0001$ & $<0.0001$ & 0.0323 \\
\hline & LAGB & 39.03 & 36.18 & 33.19 & 29.81 & 27.69 & 24.73 \\
\hline Obstructive & BPD/DS & 60.79 & 55.11 & 45.85 & 35.7 & 38.46 & 30.5 \\
\hline Sleep & LRYGB & 48.03 & 41.59 & 32.26 & 25.22 & 22.4 & 19.88 \\
\hline \multirow[t]{4}{*}{ Apnea } & RYGB & 49.99 & 45.99 & 38.23 & 30.98 & 28.55 & 24.86 \\
\hline & SG & 42.98 & 37.75 & 32.14 & 26.05 & 27.69 & 18.29 \\
\hline & $\mathrm{P}$ value & $<0.0001$ & $<0.0001$ & $<0.0001$ & $<0.0001$ & $<0.0001$ & $<0.0001$ \\
\hline & LAGB & I.55 & 1.45 & 1.56 & $|.5|$ & 1.68 & 1.8 \\
\hline
\end{tabular}


Table Continued...

\begin{tabular}{llllllll}
\hline \multicolumn{1}{l}{ Cardiopulmonary } \\
\hline & co-morbidities (\%) & & & & \\
Months: & Baseline & $\mathbf{2}$ & $\mathbf{6}$ & $\mathbf{I 2}$ & $\mathbf{1 8}$ & $\mathbf{2 4}$ \\
\hline Hypoventilation & BPD/DS & 2.09 & 1.54 & 1.14 & 0.65 & 0.43 & 0 \\
Syndrome & LRYGB & 1.88 & 1.59 & 1.36 & 1.32 & 1.35 & 1.38 \\
& RYGB & 3.32 & 2.73 & 1.91 & 1.48 & 0.97 & 0.72 \\
& SG & 1.43 & 1.31 & 1.59 & 1.74 & 2.77 & 2.44 \\
& P value & $<0.0001$ & $<0.0001$ & 0.0203 & 0.1189 & 0.0384 & 0.0375 \\
& LAGB & 15.21 & 14.35 & 13.6 & 13.16 & 12.76 & 11.94 \\
& BPD/DS & 23.07 & 19.03 & 18.68 & 19.39 & 14.53 & 11.35 \\
& LRYGB & 18.72 & 16.81 & 15.25 & 13.91 & 13.99 & 13.2 \\
& RYGB & 20.52 & 18.71 & 17.24 & 15.15 & 16.89 & 15.03 \\
& SG & 15.44 & 14.23 & 13.77 & 14.47 & 16.31 & 16.46 \\
& P value & $<0.0001$ & $<0.0001$ & $<0.0001$ & $<0.0001$ & 0.0014 & 0.0244 \\
\hline
\end{tabular}

Table 3 Metabolic and endocrine baseline and post-operative outcomes by operation

\begin{tabular}{|c|c|c|c|c|c|}
\hline \multicolumn{6}{|c|}{ Metabolic and endocrine co-morbidities (\%) } \\
\hline & Months: & 2 & 6 & 18 & 24 \\
\hline & \multicolumn{5}{|l|}{ Operation } \\
\hline & LAGB & 25.38 & 22.96 & 20.03 & 18.54 \\
\hline Diabetes & BPD/DS & 26.17 & 18.02 & 12.82 & 9.22 \\
\hline \multirow[t]{6}{*}{ Mellitus } & LRYGB & 27.46 & 19.43 & 12.69 & 11.36 \\
\hline & RYGB & 30.42 & 23.95 & 18.35 & 16.33 \\
\hline & SG & 24.13 & 18.29 & 15.69 & 10.37 \\
\hline & $P$ value & $<0.0001$ & $<0.0001$ & $<0.0001$ & $<0.0001$ \\
\hline & LAGB & 2.88 & 2.21 & 2 & 2.35 \\
\hline & BPD/DS & 4.56 & 4 & 5.56 & 4.96 \\
\hline \multirow[t]{6}{*}{ Gout } & LRYGB & 3.25 & 2.8 & 2.36 & 2.03 \\
\hline & RYGB & 3.66 & 3.16 & 4.13 & 3.47 \\
\hline & SG & 3.33 & 2.87 & 2.46 & 1.83 \\
\hline & $P$ value & $<0.0001$ & $<0.0001$ & $<0.0001$ & 0.0317 \\
\hline & LAGB & 37.23 & 35.86 & 33.17 & 30.85 \\
\hline & BPD/DS & 35.53 & 28.12 & 14.53 & 14.18 \\
\hline \multirow[t]{5}{*}{ Hyperlipidemia } & LRYGB & 38.11 & 31.83 & 24.76 & 23.35 \\
\hline & RYGB & 35.57 & 30.93 & 27.1 & 26.01 \\
\hline & SG & 34.81 & 31.97 & 25.54 & 25 \\
\hline & $P$ value & $<0.0001$ & $<0.0001$ & $<0.0001$ & $<0.0001$ \\
\hline & LAGB & 4.34 & 4.23 & 4.06 & 3.95 \\
\hline Polycystic & BPD/DS & 6.71 & 6.01 & 3.42 & 4.96 \\
\hline Ovarian & LRYGB & 5.05 & 4.51 & 3.97 & 3.34 \\
\hline \multirow[t]{4}{*}{ Syndrome } & RYGB & 3.74 & 3.64 & 3.4 & 2.75 \\
\hline & SG & 4.49 & 3.49 & 4.31 & 1.22 \\
\hline & $P$ value & $<0.0001$ & 0.0002 & 0.8824 & 0.0688 \\
\hline & LAGB & 1.07 & 0.96 & 0.77 & 0.81 \\
\hline
\end{tabular}

Citation: Finley C, Kushnir L, Slotman GJ. Outcomes variation in weight and resolution of obesity co-morbidities between five bariatric operations. Adv Obes Weight Manag Control. 2020; I0(4):I 17-127. DOI: 10.15406/aowmc.2020.10.003I7 
Table Continued...

\begin{tabular}{llllll}
\hline \multicolumn{7}{l}{ Metabolic and endocrine co-morbidities (\%) } \\
\hline \multicolumn{7}{l}{ Months: } & $\mathbf{2}$ & $\mathbf{6}$ & $\mathbf{1 8}$ & $\mathbf{2 4}$ \\
\hline Pseudotumor & BPD/DS & 1.05 & 0.48 & 0.43 & 0.71 \\
Cerebri & LRYGB & 1.91 & 1.48 & 1.71 & 1.85 \\
& RYGB & 1.86 & 1.16 & 1.09 & 1.45 \\
& SG & 0.84 & 0.74 & 1.23 & 1.22 \\
& P value & $<0.0001$ & $<0.0001$ & $<0.0001$ & $<0.0001$ \\
\hline
\end{tabular}

Outcomes for hepatobiliary diseases are shown in Table 4. LAGB: At baseline and 24 months post-operatively, patients undergoing LAGB had the lowest frequencies of liver disease, cholelithiasis, abdominal hernias, and panniculitis. BPD/DS: The BPD/DS group recorded the highest proportion of pre- and postoperative abdominal hernias, symptomatic abdominal skin, GERD, and urinary incontinence as well as the most postoperative cholelithiasis. Liver disease, LRYGB, and hernias, symptomatic panniculus, and urinary incontinence were second lowest. RYGB: Liver disease was highest, both pre- and postoperatively. Post-operative hernia and abdominal panniculitis were second highest. SG: Liver disease and urinary incontinence were least frequent after SG, while cholelithiasis and GERD were second most common.

Table 4 Hepatobiliary and abdominal baseline and post-operative outcomes by operation

\begin{tabular}{|c|c|c|c|c|c|c|}
\hline \multicolumn{7}{|c|}{ Hepatobiliary and abdominal co-morbidities (\%) } \\
\hline & Months: & Baseline & 2 & 12 & 18 & 24 \\
\hline & \multicolumn{6}{|l|}{ Operation } \\
\hline & LAGB & 4.22 & 4.02 & 3.48 & 3.51 & 3.2 \\
\hline Liver & $\mathrm{BPD} / \mathrm{DS}$ & 6.16 & 9.61 & 9.37 & 9.83 & 8.51 \\
\hline \multirow[t]{6}{*}{ Disease } & LRYGB & 7.18 & 6.99 & 6.45 & 6.78 & 4.48 \\
\hline & RYGB & 11.19 & 12.61 & 11.62 & 9.6 & 10.69 \\
\hline & SG & 4.94 & 4.71 & 4.34 & 4.62 & 3.05 \\
\hline & $\mathrm{P}$ value & $<0.0001$ & $<0.0001$ & $<0.000$ I & $<0.0001$ & $<0.0001$ \\
\hline & LAGB & 16.83 & 16.48 & 17.15 & 17.74 & 17.59 \\
\hline & BPD/DS & 21.76 & 32.33 & 45.72 & 53.42 & 50.35 \\
\hline \multirow[t]{5}{*}{ Cholelithiasis } & LRYGB & 21.58 & 21.31 & 22.67 & 23.19 & 22.53 \\
\hline & RYGB & 19.37 & 18.59 & 19.36 & 17.13 & 19.22 \\
\hline & SG & 18.18 & 17.92 & 21.13 & 25.23 & 27.44 \\
\hline & $P$ value & $<0.0001$ & $<0.0001$ & $<0.0001$ & $<0.0001$ & $<0.0001$ \\
\hline & LAGB & 4.52 & 4.35 & 4.29 & 4.21 & 4.41 \\
\hline Abdominal & BPD/DS & 10.46 & 9.54 & 23.59 & 35.04 & 27.66 \\
\hline \multirow[t]{5}{*}{ Hernia } & LRYGB & 5.09 & 4.94 & 5.11 & 4.79 & 5.37 \\
\hline & RYGB & 7.66 & 7.15 & 7.98 & 9.72 & 10.12 \\
\hline & SG & 6.31 & 5.92 & 9.33 & 11.69 & 9.15 \\
\hline & $P$ value & $<0.001$ & $<0.001$ & $<0.000 \mathrm{I}$ & $<0.0001$ & $<0.0001$ \\
\hline & LAGB & 4.54 & 4.19 & 4.4 & 4.5 & 4.08 \\
\hline Abdominal & BPD/DS & 20.44 & 16.56 & 23.75 & 16.67 & 16.31 \\
\hline Skin and & LRYGB & 7.66 & 7.31 & 8.42 & 9.79 & 7.24 \\
\hline Panniculus & RYGB & 8.03 & 7.84 & 9.37 & 8.63 & 10.55 \\
\hline
\end{tabular}


Table Continued...

\begin{tabular}{|c|c|c|c|c|c|c|}
\hline \multicolumn{7}{|c|}{ Hepatobiliary and abdominal co-morbidities (\%) } \\
\hline & Months: & Baseline & 2 & 12 & 18 & 24 \\
\hline \multirow[t]{3}{*}{ Symptoms } & SG & 8.04 & 7.37 & 9.77 & 14.77 & 9.15 \\
\hline & $P$ value & $<0.0001$ & $<0.0001$ & $<0.0001$ & $<0.0001$ & $<0.0001$ \\
\hline & LAGB & 42.44 & 33.24 & 26.17 & 25.81 & 24.66 \\
\hline Gastro & BPD/DS & 50.75 & 44.7 & 35.7 & 38.89 & 36.88 \\
\hline Esophageal & LRYGB & 49.74 & 37.79 & 24.27 & 23.59 & 21.95 \\
\hline Reflux & RYGB & 42.29 & 32.78 & 26 & 26.49 & $28.6 \mathrm{I}$ \\
\hline \multirow[t]{3}{*}{ Disease } & SG & 42.98 & 37.71 & 35.11 & 35.08 & 32.93 \\
\hline & $P$ value & $<0.0001$ & $<0.0001$ & $<0.0001$ & $<0.0001$ & $<0.0001$ \\
\hline & LAGB & 24.66 & 18.2 & 15.86 & 15.33 & $|4.6|$ \\
\hline Urinary & BPD/DS & 36.88 & 29.43 & 22.62 & 27.35 & 21.28 \\
\hline \multirow[t]{4}{*}{ Incontinence } & LRYGB & 21.95 & 19.59 & 14.42 & 13.93 & 13.37 \\
\hline & RYGB & 28.61 & 16.69 & 13.48 & 13.37 & 13.29 \\
\hline & SG & 32.93 & 17.18 & 13.75 & 15.69 & 8.54 \\
\hline & $P$ value & $<0.0001$ & $<0.0001$ & $<0.0001$ & $<0.0001$ & 0.0053 \\
\hline
\end{tabular}

Somatic disorder outcomes are displayed in Table 5. LAGB: All somatic complaints were reported least commonly by LAGB patients at baseline and were second lowest poster operatively. BDP/DS: Patients undergoing BPD/DS complained the most in all somatic disorders preoperatively. By 24 months postoperatively, lower extremity edema and musculoskeletal pain continued to occur most commonly and back pain was also second highest. LRYGB: All somatic disorders were lowest postoperatively in the LRYGB patients. RYGB: Postoperative back pain afflicted RYGB patients most commonly. SG: The SG group had the second most frequent rate of postoperative lower extremity edema.

Table 5 Somatic disorders baseline and post-operative outcomes by Operation

\begin{tabular}{|c|c|c|c|c|c|c|c|}
\hline \multicolumn{8}{|c|}{ Somatic co-morbidities (\%) } \\
\hline & Months: & Baseline & 2 & 6 & 12 & 18 & 24 \\
\hline & \multicolumn{7}{|l|}{ Operation } \\
\hline & LAGB & 44.57 & 40.08 & 36.84 & 33.76 & 32.01 & 30.03 \\
\hline Back & BPD/DS & 60.49 & 51.54 & 45.95 & 41.2 & 39.74 & 39.72 \\
\hline \multirow[t]{5}{*}{ Pain } & LRYGB & 49.88 & 43.25 & 36.3 & 32.59 & 29.45 & 29.56 \\
\hline & RYGB & 50.73 & 46.24 & 41.72 & 40.58 & 46.42 & 41.33 \\
\hline & SG & 44.56 & 37.98 & 34.27 & 31.48 & 31.69 & 33.54 \\
\hline & $P$ value & $<0.000$ I & $<0.000$ I & $<0.000$ I & $<0.000$ I & $<0.000$ I & $<0.0001$ \\
\hline & LAGB & 23.09 & 18.94 & 17.09 & 15.87 & 15.35 & 13.49 \\
\hline Lower & BPD/DS & 47.88 & 40.46 & 37.85 & 37.48 & 41.03 & 32.62 \\
\hline Extremity & LRYGB & 30.19 & 22.28 & $17.4 \mid$ & 15.1 & 14.34 & 13.07 \\
\hline \multirow[t]{4}{*}{ Edema } & RYGB & 26.65 & 20.91 & 19.24 & 18.26 & 16.52 & $|4.3|$ \\
\hline & SG & 27.59 & 21.22 & 19.79 & 18.6 & 21.54 & 20.73 \\
\hline & $P$ value & $<0.0001$ & $<0.0001$ & $<0.0001$ & $<0.0001$ & $<0.0001$ & $<0.0001$ \\
\hline & LAGB & 38.74 & 33.84 & 31.82 & 28.7 & 27.92 & 26.28 \\
\hline Musculo & BPD/DS & 49.67 & 43.6 & 38.99 & 37.16 & 36.32 & 32.62 \\
\hline Skeletal & LRYGB & 46.42 & 39.02 & 33.18 & 29.61 & 26.94 & 26.13 \\
\hline \multirow[t]{3}{*}{ Pain } & RYGB & 39.8 & 35.22 & 32.39 & 30.74 & 30.62 & $28.6 \mathrm{I}$ \\
\hline & SG & 39.1 & 31.86 & 30.1 & 28.36 & 29.23 & 28.66 \\
\hline & $P$ value & $<0.0001$ & $<0.0001$ & $<0.0001$ & $<0.0001$ & 0.0055 & 0.2629 \\
\hline
\end{tabular}

Citation: Finley C, Kushnir L, Slotman GJ. Outcomes variation in weight and resolution of obesity co-morbidities between five bariatric operations. Adv Obes Weight Manag Control. 2020; I0(4):I 17-127. DOI: 10.15406/aowmc.2020.10.003I7 
Table 6 demonstrates outcomes in psychological and behavioral disorders. LAGB: LAGB patients had the lowest frequency of psychological disorders at baseline and post-operatively. BPD/DS: Patients who underwent BPD/DS suffered from all psychological disorders the most at baseline and postoperative mental health, depression, and psychological impairment diagnoses continued to be the highest. BPD/DS patients were the only group to have worsening postoperative mental health diagnosis and psychological impairment. Alcohol use postoperatively was also reported most in the BPD/DS group. LRYGB: The LRYGB group mirrored the LAGB group for the least impaired functional status. RYGB: Impaired functional status was most frequent postoperatively after RYGB, but postoperative alcohol use was least. SG: Depression was diagnosed least frequently at 24 months after SG.

Table 6 Psychological and behavioral baseline and post-operative outcomes by operation

\begin{tabular}{|c|c|c|c|c|c|c|c|}
\hline \multicolumn{8}{|c|}{ Psychological and behavioral disorders (\%) } \\
\hline & Months: & Baseline & 2 & 6 & 12 & 18 & 24 \\
\hline & \multicolumn{7}{|c|}{ Operation } \\
\hline & LAGB & 8.88 & 8.49 & 8.04 & 7.4 & 7.38 & 6.72 \\
\hline Mental & BPD/DS & 11.84 & 11.21 & 12.11 & 12.6 & 14.53 & 14.89 \\
\hline Health & LRYGB & 11.35 & 10.87 & 9.92 & 9.7 & 9.83 & 10.35 \\
\hline \multirow[t]{4}{*}{ Diagnosis } & RYGB & 11.34 & 10.79 & 10.11 & 9.37 & 8.99 & 9.97 \\
\hline & SG & 10.66 & 10.26 & 9.97 & 9.7 & 11.08 & 9.76 \\
\hline & $P$ value & $<0.0001$ & $<0.0001$ & $<0.0001$ & $<0.0001$ & $<0.0001$ & $<0.0001$ \\
\hline & LAGB & 2.13 & 1.89 & 1.79 & 1.71 & 1.7 & 1.74 \\
\hline Impaired & BPD/DS & 7.17 & 5.97 & 6.29 & 4.52 & 5.56 & 4.96 \\
\hline Functional & LRYGB & 3.35 & 2.88 & 2.56 & 2.36 & 2.27 & 1.77 \\
\hline \multirow[t]{5}{*}{ Status } & RYGB & 5.77 & 5.38 & 5.46 & 5.21 & 6.56 & 6.36 \\
\hline & SG & 3.2 & 2.65 & 2.7 & 2.53 & 1.54 & 5.49 \\
\hline & $P$ value & $<0.0001$ & $<0.0001$ & $<0.0001$ & $<0.0001$ & $<0.0001$ & $<0.0001$ \\
\hline & LAGB & 31.42 & 30.06 & 29.46 & 29.14 & 28.98 & 28.39 \\
\hline & BPD/DS & 40.47 & 36.58 & 34.7 & 35.86 & 35.04 & 38.3 \\
\hline \multirow[t]{5}{*}{ Depression } & LRYGB & 36.65 & 34.2 & 32.17 & 31.38 & 30.76 & 32.39 \\
\hline & RYGB & 33.44 & 31.82 & 30.63 & 30.5 & 27.22 & 30.06 \\
\hline & SG & 33.37 & 30.85 & 31.06 & 29.52 & 33.23 & 26.83 \\
\hline & $P$ value & $<0.0001$ & $<0.0001$ & $<0.0001$ & $<0.0001$ & 0.0063 & $<0.0001$ \\
\hline & LAGB & 13.82 & 13.28 & $|2.7|$ & 11.73 & 11.77 & 11.23 \\
\hline Pyschological & BPD/DS & 16.08 & 14.47 & 15.44 & 15.99 & 17.52 & 20.57 \\
\hline \multirow[t]{5}{*}{ Impairment } & LRYGB & 17.77 & 16.85 & $|5.4|$ & 14.92 & 15.15 & 15.42 \\
\hline & RYGB & 17.83 & 16.89 & 15.93 & 15.34 & 14.95 & 15.9 \\
\hline & SG & 16.69 & 16.04 & 15.56 & 14.62 & 15.69 & 15.24 \\
\hline & $P$ value & $<0.0001$ & $<0.0001$ & $<0.0001$ & $<0.0001$ & $<0.0001$ & $<0.0001$ \\
\hline & LAGB & 30.77 & 26.87 & $25.7 I$ & 24.17 & 23.14 & 22.69 \\
\hline Alcohol & BPD/DS & 34.67 & 26.97 & 26.88 & 28.76 & 28.63 & 32.62 \\
\hline \multirow[t]{5}{*}{ Abuse } & LRYGB & 30.96 & 22.46 & 21.52 & 21.54 & 20.22 & 21.52 \\
\hline & RYGB & 15.85 & 12.28 & 12.73 & 12.33 & 10.45 & 11.42 \\
\hline & SG & 34.73 & 1.52 & 26.83 & 25.62 & 19.08 & 20.73 \\
\hline & $P$ value & $<0.0001$ & $<0.000$ I & $<0.0001$ & $<0.0001$ & $<0.0001$ & $<0.0001$ \\
\hline & LAGB & 0.37 & 0.35 & 0.29 & 0.26 & 0.15 & 0.14 \\
\hline Substance & BPD/DS & 0.9 & 0.62 & 0.95 & 1.29 & 1.71 & 0.71 \\
\hline
\end{tabular}




\begin{tabular}{|c|c|c|c|c|c|c|c|}
\hline \multicolumn{8}{|c|}{ Psychological and behavioral disorders (\%) } \\
\hline & Months: & Baseline & 2 & 6 & 12 & 18 & 24 \\
\hline & \multicolumn{7}{|c|}{ Operation } \\
\hline \multirow[t]{5}{*}{ Abuse } & LRYGB & 0.45 & 0.38 & 0.39 & 0.37 & 0.35 & 0.32 \\
\hline & RYGB & 0.28 & 0.29 & 0.3 & 0.33 & 0.73 & 0.72 \\
\hline & SG & 0.28 & 0.26 & 0.28 & 0.51 & 0.31 & 1.83 \\
\hline & $P$ value & 0.0002 & 0.1522 & 0.0009 & $<0.0001$ & $<0.0001$ & $<0.0001$ \\
\hline & LAGB & 6.44 & 5.74 & 5.18 & 4.5 & 4.22 & 4.18 \\
\hline Tobacco & BPD/DS & 6.16 & 4.74 & 5.62 & 6.14 & 7.26 & 7.09 \\
\hline \multirow[t]{4}{*}{ Use } & LRYGB & 6.62 & 5.25 & 4.93 & 4.53 & 4.06 & 4.83 \\
\hline & RYGB & 5.55 & 4.8 & 4.12 & 4.25 & 4.37 & 4.91 \\
\hline & SG & 7.25 & 5.89 & 5.48 & 5.14 & 4.31 & 6.1 \\
\hline & $P$ value & 0.001 & $<0.0001$ & 0.0239 & 0.2569 & 0.2087 & 0.1468 \\
\hline
\end{tabular}

\section{Discussion}

The results of this investigation identified statistically and clinically significant variations in post-operative weight loss and resolution of obesity-related morbidities among the five bariatric operations evaluated. BPD/DS induced the most profound reduction in BMI, followed by RYGB and LRYGB. Hypertension resolved best with BPD/DS. The incidence of OSA was reduced most effectively with SG while asthma resolved most after BPD/DS. BPD/DS patients had the most resolution of diabetes, although SG and LRYGB were only slightly less successful. There was a clear advantage in hyperlipidemia resolution after BPD/DS. GERD symptoms improved most after LRYGB. BPD/DS patients developed hepatobiliary and abdominal wall disorders most dramatically. While LAGB patients had the lowest weight and BMI preoperatively as well as the fewest obesity comorbidities, weight/BMI reduction and resolution of weight-related medical problems responded significantly less following LAGB. Our review of the literature indicates that this wide variation of bariatric surgery outcomes, comparing results from the five most frequently performed procedures in the largest population to date, has not been reported previously, and is an important finding of this study.

The malabsorptive operations, especially BPD/DS, outperformed the restrictive procedures in lowering BMI and weight, consistent with previous reports that compared $\mathrm{BPD} / \mathrm{DS}$ to other bariatric procedures. ${ }^{14-17}$ Due to its ability to induce massive weight loss, authors argue that BPD/DS offers the most benefit in treating the superobese (BMI $>50$ ), a subset of patients among whom success has not been as consistent after other bariatric surgeries. In the present study, both Roux-en-Y patient groups experienced greater BMI reduction than the restrictive procedures, although these results were not dramatically better than with SG. Several studies have investigated the outcomes in weight loss between RYGB/LRYGB and SG and with inconsistent findings. ${ }^{10,12}$ Our large population analysis of 2 year outcomes revealed a slight advantage after gastric bypass over SG. LAGB had the least effect on weight illustrating why its use is generally avoided in cases where higher reduction in excess body mass is desired. ${ }^{18}$

The success in BMI reduction correlated with resolution of hypertension. BPD/DS resulted in a dramatic reduction from the highest incidence of hypertensive at baseline to the lowest by two years. Similarly, BPD/DS patients transitioned from the highest BMI group to the lowest, suggesting a causal relationship between loss of excess body mass and the successful resolution of hypertension, as has been described previously. ${ }^{19,20}$ Success in treatment of hypertension directly paralleled weight loss for all groups except for RYGB. No previous reports have found similar differences between open and laparoscopic RY outcomes. BMI and weight appeared to be unrelated to resolution of other cardiovascular obesity co-morbidities. It is possible the benefits from weight reduction on blood pressure control may not become clinically apparent within 2 years and further investigations may be needed for longer term analysis.

Obesity-related pulmonary diseases resolved with varying success between the operations. Pre-operatively, in this investigation, the incidence of OSA was directly proportional to the mean BMI of patients who chose BPD/DS, RYGB, LRYGB, SG or LAGB. Weight loss has effectively treated OSA. ${ }^{21}$ In the present study, however, while OSA resolved well after all five bariatric procedures, BMI did not relate numerically to OSA resolution. BDP/DS patients had the highest OSA in spite of achieving the lowest BMI at two years, while SG and LRYGB resolved OSA best, even with less dramatic BMI outcomes.

Prior studies have identified obesity as a risk factor for asthma and weight loss as an effective form of asthma management. ${ }^{22}$ This association was evident in the current investigation, where the baseline prevalence of asthma was associated directly with the relative BMI for each group. As with hypertension, BPD/DS most effectively reduced the frequency of asthma, whose patients once again transitioned from having the most to least asthmatics over the two year observation period. AGB was also effective in treatment of asthma, although there was a much less overall resolution from an already low baseline asthmatic rate. SG was the only operation that worsened asthma, a finding that has been described by other sources, citing increasing GERD symptoms as the likely etiology. ${ }^{23}$

Outcomes in resolution of diabetes did not demonstrate any definitive advantage between malabsorptive and restrictive procedures. The connection between obesity and type 2 diabetes has been well established and reduction in excess body mass predominates as the most effective treatment. ${ }^{24} \mathrm{BPD} / \mathrm{DS}$ most effectively treated diabetes and, again, the direct link between excess weight and diabetes was apparent in the BPD/DS group who transitioned from the most to least 
diabetic patient group. Similar to the outcomes observed here, previous investigations have reported $\mathrm{BPD} / \mathrm{DS}$ to have higher resolution rates of diabetes than other bariatric surgeries when compared directly. ${ }^{13,14}$ SG slightly outperformed the LRYGB group while open RYGB was much less successful. Although SG patients presented less often with diabetes at baseline than the gastric bypass groups, SG and LRYGB had a very similar relative reduction from baseline rates while SG performed numerically better then RYGB. This directly contradicts previous clinical trials in which gastric bypass regularly outperformed restrictive procedures in treatment of diabetes. ${ }^{9-12}$ Researchers have proposed that intestinal malabsorption is a better mechanism for diabetic control due to more limited intestinal absorption of glucose and free fatty acids, advantages from altered hormonal activity, and more mediating effects from altered foregut/hindgut anatomy. ${ }^{9}$ Our investigation indicates that although $\mathrm{BPD} / \mathrm{DS}$ resolved diabetes most effectively, the previously reported advantage in malabsorptive procedures over restrictive procedures cannot be confirmed presently. Also, degree of body mass reduction does not necessarily correlate directly with the reduced post-operative prevalence of diabetes. Further investigation is still needed to identify the causal mechanism of diabetic control after different bariatric procedures.

The strong association of atherogenic dyslipidemia with other obesity-related co-morbidities was evident from outcomes analyzed in this study. The interrelation of hyperlipidemia, hypertension, elevated fasting glucose, and central obesity comprise metabolic syndrome and increase the risk of diabetes and cardiovascular disease. These disorders also occur more commonly together than by chance alone. ${ }^{25}$ In the present investigation, baseline characteristics for each group demonstrated this association between the disorders of metabolic syndrome: baseline BMI corresponded in the distribution of HTN, DM, and hyperlipidemia. The post-operative outcomes reported here demonstrate that reducing excess body mass and central obesity has an immediate and reciprocal impact on the other components of metabolic syndrome. BPD/DS patients had the most significant reduction in BMI and subsequently the most profound resolution of hyperlipidemia as well as diabetes and hypertension. Furthermore, the relative success in treatment of the adjoined disorders defining metabolic syndrome directly correlated with the relative degree of BMI reduction for each operation. The RYGB group was the only exception. RYGB patients experienced the second most significant reduction in BMI, but resolution of HTN, DM, and dyslipidemia was among the least, finishing only ahead of LAGB patients. All previous reports found open gastric bypass to be equally as effective as laparoscopic gastric bypass in treating these disorders. ${ }^{26}$ However, from the BOLD outcomes evaluated here, open gastric bypass is far less efficacious at treating the disorders of metabolic syndrome than LRYGB, despite equivocal weight loss. The mechanism behind this finding is not clear from the data.

Although obesity is a known independent risk factor for reflux disease, higher reduction in BMI did not directly correlate with effective GERD treatment. Gastric bypass has long been the most effective bariatric procedure for resolving reflux disease, due to anatomical diversion of gastric acid, and is even offered as a revisional option after failed antireflux surgery. ${ }^{27}$ These findings were confirmed in our results, with LRYGB patients experiencing the greatest resolution of GERD. RYGB was less successful than LRYGB indicating a superior effect of the laparoscopic procedure in treating reflux. BPD/DS patients received the least benefit in their reflux symptoms, indicating that weight loss was not an independent factor for GERD resolution and/or the difference in the anatomical reconstruction of the foregut between gastric bypass and BPD/DS influenced GERD resolution. Although SG was less effective in GERD treatment, the SG patients did experience improvement in GERD rates. The BOLD outcomes in this investigation revealed that the prevalence of GERD dd not increase after SG, which phenomenon continues to be a topic of investigation. ${ }^{28,29}$

Evaluation of post-operative abdominal and hepatobiliary diseases revealed how more invasive procedures, while achieving better weight loss results, carry increased risks of postoperative morbidity. Pre-existing liver disease is common in bariatric patients. Liver disease among the patients in this report varied directly with baseline BMI. Post-operatively, however, only BPD/DS patients experienced increased liver disease. Liver impairment after BPD/DS has long been investigated as a possible complication secondary to rapid weight loss and protein malnutrition. Keshishian et al studied repeated liver biopsies in post-operative BPD/DS patients and found increased hepatic inflammation at 6 months with gradual improvements after 12 months and eventually $60 \%$ improvement of steatosis by 3 years. ${ }^{30}$ Similar findings occurred in our BPD/DS group, possibly indicating a post-operative inflammatory response after BPD/DS.

Cholelithiasis is another hepatobiliary disease thought to be affected by the massive weight loss after bariatric surgery. ${ }^{31,32}$ Cholelithiasis in bariatric patients, particularly those undergoing bypass procedures, has been of particular concern and the need for prophylactic cholecystectomy continues to be unclear. ${ }^{33-35}$ Our analysis found that gallstone rates were unaffected by bariatric surgery except those undergoing $\mathrm{BPD} / \mathrm{DS}$ and $\mathrm{SG}$. The massive rise in cholelithiasis after BPD/DS would seem to confirm the theory that gallstone formation is affected by massive weight loss, but the unaffected rates of the bypass groups challenge that notion. Thus, the propensity for gallstone formation after BPD/DS may be more likely inherent to the biliary diversion. Incisional hernias add morbidity and additional operations to bariatric surgery. Hernia risks are increased in open surgeries compared to laparoscopic. ${ }^{36}$ This increased risk was confirmed in this study, as the incidence of hernias developing after $\mathrm{BPD} / \mathrm{DS}$ and RYGB was highest. However, according to an analysis of BOLD by Nelson et al, 50\% of BPD/DS surgeries in BOLD were performed laparoscopically. ${ }^{37}$ The outcome data was not delineated between the laparoscopic and open BPD/DS patients, therefore the marked increase in hernia development after BPD/DS cannot be safely attributed to laparotomy. Also concerning the abdominal wall is panniculitis, which presumably would be directly dependent to degree of weight loss and excess abdominal skin. This concept was partly evident here, with the most symptoms developing after BPD/ DS and RYGB, but LRYGB patients also had significant weight loss with no subsequent rise in pannicular symptoms. The added insult to the abdominal skin in the open surgeries may lead to increased risks for pannicular symptoms over laparoscopic surgeries that induce equivocal weight loss, although outcomes after laparoscopic and open $\mathrm{BPD} / \mathrm{DS}$ patients would allow more definitive evidence.

There are a number of limitations in this review. This was a retrospective analysis, although data was collected prospectively. Each group consisted of self-selected patients seeking to undergo a desired operation which could lead to bias. The attrition rate over two years did increase, but our analysis still revealed statistically significant findings and the power of this study at two years was still significant. Another limitation was that the classification of each co-morbidity was defined by BOLD clinical criteria. For example, since liver biopsy was not standardized in BOLD, liver disease was diagnosed only clinically. BPD/DS outcomes were not differentiated by laparoscopic and open operations which limited evaluation. 


\section{Conclusion}

Our investigation confirmed that statistically and clinically significant variations exist in postoperative weight loss and obesityrelated co-morbidities between the five operations evaluated in this study. Understanding of the relative benefits and problems of each operation can allow for more effective, individualized treatment of obesity and obesity-associated diseases. BPD/DS is the most effective surgical modality in reduction of excessive body mass as well as resolution of several major com-morbidities including diseases related to metabolic syndrome and asthma. However, $\mathrm{BPD} / \mathrm{DS}$ is least effective in treating OSA, GERD, musculoskeletal complaints, and urinary incontinence. Post-operatively, BPD/DS patients are at the highest risk for worsening liver disease, gallstones, hernias, panniculitis, and psychological/substance abuse disorders. Patients undergoing LRYGB have the best relief of GERD and musculoskeletal complaints, while also benefitting from the second highest reduction in weight. Overall, LRYGB patients consistently experienced resolution of nearly every co-morbidity. Although open RYGB is comparable to laparoscopic for weight loss, the open procedure does not treat related co-morbidities as successfully. Sleeve gastrectomy induces slightly less weight loss than the malabsorptive operations, but has the best post-operative reduction in OSA, gout, liver disease, and depression. SG and LRYGB had comparable outcomes in resolution of hypertension, diabetes, and hyperlipidemia. AGB has the least impact on weight loss and most major obesityrelated diseases, but was least likely to experience worsening postoperative hernias, liver dysfunction, cholelithiasis, and panniculitis. These previously unreported findings may add to clinical judgment of bariatric surgeons and facilitate optimized management of fragile morbidly obese patients.

\section{Acknowledgments}

None.

\section{Conflicts of interest}

There were no potential conflicts of interest in this analysis.

\section{Funding}

This research did not receive grant funding from the public, commercial, or not-for-profit sectors.

\section{References}

1. Padwal R, Li SK, Lau DC. Long-term pharmacotherapy for obesity and overweight. Int J ObesRelat Metab Disord. 2003;27(12):1437-1446.

2. Brennan L, Walkley J, Fraser SF, et al. Motivational interviewing and cognitive behaviour therapy in the treatment of adolescent overweight and obesity: study design and methodology. Contemp Clin Trials. 2008;29(3):359-375.

3. Shaw K, O'Rourke P, Del Mar C, et al. Psychological interventions for overweight or obesity. Cochrane Database Syst Rev. 2005;(2):CD003818.

4. Armstrong MJ, Mottershead TA, Ronksley PE, et al. Motivational interviewing to improve weight loss in overweight and/or obese patients: a systematic review and meta-analysis of randomized controlled trials. JAMA. 2005;294(15):1909-1917.

5. Trus TL, Pope GD, Finlayson SR. National trends in utilization and outcomes of bariatric surgery. Surg Endosc. 2005;19:616-620.

6. Sages Guideline Committee/ASMBS. Guidelines for clinical application for laparoscopic bariatric surgery. 2008.
7. Santry HP, Gillen DL, Lauderdale DS. Trends in Bariatric Surgical Procedures. JAMA. 2005;294(15):1909-1917.

8. Pope GD, Birkmeyer JD, Finlayson SR. National trends in utilization and in-hospital outcomes of bariatric surgery. $J$ Gastrointest Surg. 2002;6:855-860.

9. Lee WJ, Chong K, Kong Han S, et al. Gastric bypass vs gastric sleeve for type 2 diabetes mellitus: a randomized control trial. Arch Surg. 2011;146(2):143-148.

10. Peterli R, Wölnerhanssen BK, Vetter D, et al. Laparoscopic sleeve gastrectomy versus Roux-en-Y gastric bypass for morbid obesity 3 year outcomes of the prospective randomized swiss multicenter bypass or sleeve study (SM-BOSS). Ann of Surg. 2017;265(13):446-473.

11. Peterli R, Wölnerhanssen BK, Peters T, et al. Improvement in glucose metabolism after bariatric surgery: comparison of laparoscopic Rouxen-Y gastric bypass and laparoscopic sleeve gastrectomy: a prospective randomized trial. Ann of Surg. 2009;250(2):234-241.

12. Lakdawala MA, Bhasker A, Mulchandani D, et al. Comparison between the results of laparoscopic sleeve gastrecto my and laparoscopic Rouxen-Y gastric bypass in the Indian population: a retrospective lyear study. J Obes Surg. 2010;20(1):1-6.

13. Risstad H, Søvik TT, Engström MY, et al. Five year outcomes after laparoscopic gastric bypass and laparoscopic duodenal switch in patients with body mass index of 50 to 60 :a randomized clinical trial. JAMA. 2015;150(4):352-361.

14. Nelson DW, Kelly SB, Martin MJ. Analysis of obesity-related outcomes and bariatric failure rates with the duodenal switch vs gastric bypass for morbid obesity. Arch Surg. 2012;147(9):847-854.

15. DeMaria EJ, Pate V, Warthen M, et al. Baseline data from American Society for Metabolic and Bariatric Surgery-designated Bariatric Surgery Centers of Excellence using the Bariatric Outcomes Longitudinal Database. Surg Obes Relat Dis. 2010;6(4):347-355.

16. SAS Institute Inc. SAS/STAT® 9.22 User's Guide. Cary, NC: SAS Institute Inc; 2010.

17. Sucandy I, Titano J, Bonanni F, et al. Comparison of vertical gastric sleeve versus biliopancreatic diversion. N Am J Med Sci. 2014;6(1):35-38.

18. Tice JA, Karliner L, Walsh J, et al. Gastric banding or bypass? A systemic review comparing the two most popular bariatric procedures. Am J Med. 2008;121(10):885-893.

19. Neter JE, Stam BE, Kok FJ, et al. Influence of weight reduction on blood pressure: a meta-analysis of randomized controlled trials. Hypertension. 2003;42:878-884.

20. Noria S, Grantcharov T. Biological effects of bariatric surgery on obesityrelated comorbidities. Can J Surg. 2013;56(1):47-57.

21. Anandam A, Akinnusi M, Kufel T, et al. Effects of dietary weight loss on obstructive sleep apnea: a meta-analysis. Sleep and Breathing. 2013;17(1):227-234.

22. Eneli TS, Camargo CA. Weight loss and asthma: a systemic review. Thorax. 2008;63(8):671-676.

23. Ribo P, Pacheco A, Arrieta P, et al. Gastroesophageal reflux as a cause of chronic cough, severe asthma, and migratory pulmonary infiltrates. Respirol Case Rep. 2014;2(1):1-3.

24. Mokdad AH, Ford ES, Bowman BA. Prevalence of obesity, diabetes, and obesity-related health risk factors, 2001. JAMA. 2003;289(1);76-79.

25. Alberti KG, Eckel RH, Grundy SM, et al. Harmonizing the metabolic syndrome. Circ. 2009;120:1640-1645.

26. Nguyen NT, Ho HS, Palmer LS, et al. A comparison study of laparoscopic versus open gastric bypass for morbid obesity. $J$ Am Coll Surg. 2000;191(2):155-156. 
27. Awais O, Luketich JD, Reddy N, et al. Roux-en-Y near esophagojejunostomy for failed antireflux operations: outcomes in more than 100 patients. Ann Thor Surg. 2014;98(6):1905-1913.

28. StenardF, Ianelli A. Laparoscopic sleeve gastrectomy and gastroesophageal reflux. World J Gastroenterol. 2015;21(36):10348-10357.

29. Bohdjalian A, Langer FB, Shakeri-Leidenmühler S, et al. Sleeve gastrectomy as sole and definitive bariatric procedure: 5-year results for weight loss and ghrelin. Obes Surg. 2010;20(5):535-540.

30. Keshishian A, Zahriya K, Wiles EB. Duodenal switch has no detrimental effects on heparin function and improved steatohepatitis after 6 months. Obes Surg. 2005;15(10):1418-1423.

31. Everhart JE. Contributions of obesity and weight loss to gallstone disease. Ann Int Med. 1993;199(10):1029-1035.

32. Mummadi RR, Kasturi KS, Chennareddygari S, et al. Effect of bariatric surgery on nonalcoholic fatty liver disease: a systemic review and metaanalysis. Clin Gastr Hep. 2008;6(12):1396-1402.
33. Liem RK, Niloff PH. Prophylactic cholecystectomy with open gastric bypass operation. Obes Surg. 2004;14(6):763-765.

34. Caruana JA, McCabe MN, Smith AD, et al. Incidence of symptomatic gallstones after gastric bypass: is prophylactic treatment really necessary? Surg Obes Rel Dis. 2005;1(6):564-567.

35. D’Hondt M, Sergeant G, Deylgat B, et al. Prophylactic cholecystectomy, a mandatory step in morbidly obese patients undergoing laparoscopic Roux-en-Y gastric bypass? J Gastroint Surg. 2011;15:1532.

36. Huu RL, Mege D, Ouaissi M, et al. Incidence and prevention of ventral incisional hernia. J Visc Surg. 2012;149(5):3-14.

37. Nelson DW, Blair KS, Martin MJ. Analysis of Obesity-Related Outcomes and Bariatric Failure Rates With the Duodenal Switch vs Gastric Bypass for Morbid Obesity. Arch Surg. 2012;147(9):847-854. 